\title{
Kernos
}

Revue internationale et pluridisciplinaire de religion grecque antique

29 | 2016

Varia

\section{La complexité d'Héraclès, entre Hérodote et les cultes de Thasos}

\section{Zoé Pitz}

\section{(2) OpenEdition \\ Journals}

\section{Édition électronique}

URL : https://journals.openedition.org/kernos/2392

DOI : 10.4000/kernos.2392

ISSN : 2034-7871

\section{Éditeur}

Centre international d'étude de la religion grecque antique

\section{Édition imprimée}

Date de publication : 1 octobre 2016

Pagination : 101-118

ISSN : 0776-3824

\section{Référence électronique}

Zoé Pitz, «La complexité d'Héraclès, entre Hérodote et les cultes de Thasos », Kernos [En ligne], 29 |

2016, mis en ligne le 01 octobre 2019, consulté le 28 février 2022. URL : http://

journals.openedition.org/kernos/2392 ; DOI : https://doi.org/10.4000/kernos.2392

Ce document a été généré automatiquement le 28 février 2022.

Kernos 


\title{
La complexité d'Héraclès, entre Hérodote et les cultes de Thasos
}

\author{
Zoé Pitz
}

Je tiens à remercier chaleureusement Vinciane Pirenne-Delforge et Jan-Mathieu Carbon pour leur soutien et leurs précieux conseils. Tous mes remerciements vont également à Stella Georgoudi, dont les remarques et suggestions ont permis d'améliorer cet article.

\section{Le témoignage d'Hérodote à propos d'Héraclès}

1 À l'exception des toutes premières lignes, le livre II des Histoires consiste en une vaste digression sur l'Égypte lors de laquelle Hérodote s'intéresse successivement à la géographie du pays et aux mœurs de ses habitants ainsi qu'à l'histoire de l'Égypte, de Min à Amasis. Si la première partie est principalement descriptive, l'auteur y intègre parfois des récits, notamment des étiologies, dans le but de justifier une opinion ou d'expliquer une coutume. Ainsi, alors qu'il aborde la question des rituels sacrificiels, et en particulier celle des animaux consacrés par les Égyptiens, Hérodote rapporte le récit justifiant le fait que les habitants de Thèbes ne sacrifient jamais d'ovins, mais plutôt des caprins. Selon les Thébains eux-mêmes, Zeus, afin de se cacher d'Héraclès qui insistait pour le voir, eut l'idée d'écorcher un bélier, de se vêtir de sa toison et de tenir la tête de l'animal devant la sienne. Depuis lors, les Égyptiens donnent à la statue de Zeus une tête de bélier et considèrent cet animal comme sacré ${ }^{1}$.

Ce récit donne à Hérodote l'opportunité de démontrer que ce sont les Grecs qui ont emprunté Héraclès aux Égyptiens et non l'inverse ${ }^{2}$. Le héros Héraclès, fils d'Amphitryon, tirerait son origine d'un dieu égyptien beaucoup plus ancien. Pour étayer son propos, Hérodote fait appel à plusieurs arguments. Les trois premiers arguments relèvent de la gnômè et sont fondés tantôt sur la tradition - le premier et le troisième - , tantôt sur la probabilité - le deuxième : 1) Amphitryon et Alcmène avaient des ancêtres égyptiens. 2) Poséidon et les Dioscures sont inconnus des Égyptiens. Or, étant donné les relations maritimes entre les deux peuples, si les Égyptiens avaient emprunté quelque divinité aux Grecs, ces dieux de la mer auraient dû 
s'imposer. 3) Les Égyptiens eux-mêmes affirment qu'Héraclès est un dieu ancien. En effet, les douze dieux - dont Héraclès fait partie - seraient nés dix-sept mille ans avant le règne d'Amasis. Les quatrième et cinquième arguments proviennent d'une enquête personnelle, historiè : 4) Lorsqu'il se rendit à Tyr, parce qu'il avait entendu dire qu'il s'y trouvait un sanctuaire d'Héraclès, Hérodote discuta avec les prêtres du dieu, lesquels lui confièrent que le sanctuaire avait été établi à la même époque que la fondation de la ville, 2300 ans auparavant. 5) Lors d'un voyage à Thasos, qu'il entreprit suite à la découverte d'un deuxième sanctuaire d'Héraclès à Tyr, où le dieu était honoré sous le nom de Thasien, il vit un sanctuaire d'Héraclès, établi par les Phéniciens qui colonisèrent l'île cinq générations avant la naissance en Grèce d'Héraclès, fils d'Amphitryon ${ }^{3}$. De là, Hérodote conclut sur le passage qui nous intéresse et dont nous proposerons une traduction après analyse :

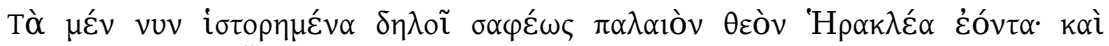

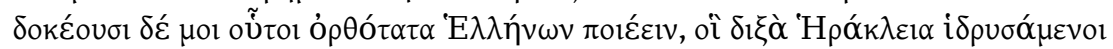

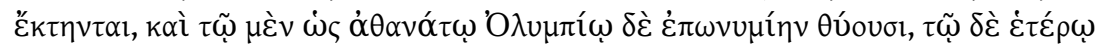

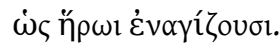

\section{Le poids des mots}

3 Composé de plusieurs termes ambigus, ce passage n'a cessé de piquer la curiosité des commentateurs d'Hérodote et des chercheurs ayant étudié le culte d'Héraclès. Il a permis d'appuyer des hypothèses plus ou moins convaincantes, notamment l'idée qu'il existait à Thasos un double culte d'Héraclès, à la fois pour le dieu et pour le héros. En revanche, trop peu d'attention a été prêtée à la compréhension fine des termes mêmes utilisés par Hérodote. Or, face à un texte si problématique et si fréquemment exploité, seule une analyse philologique minutieuse peut permettre de faire avancer la réflexion. Cette analyse examinera successivement les mots essentiels du passage que sont

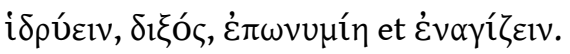

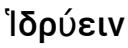

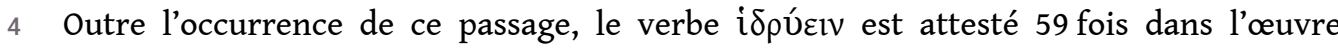
d'Hérodote. Dans 46 cas, il s'applique à un ou des substantifs faisant référence à la

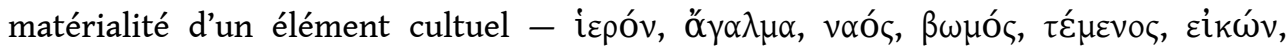
$\dot{\eta} \rho \tilde{\omega}$ ov et $\mu \alpha v \tau \varepsilon \tilde{i} v^{4}$-, signifiant ainsi sans aucun doute "fonder ", "construire",

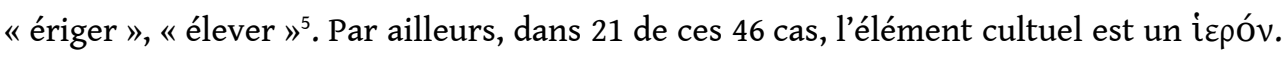

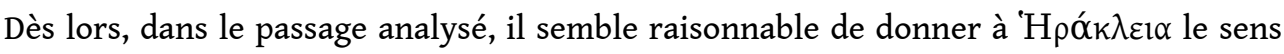
de « sanctuaires d'Héraclès » et non de «fêtes en l'honneur d'Héraclès » ${ }^{6}$.

\section{$\Delta \mathrm{l}$ ६ós}

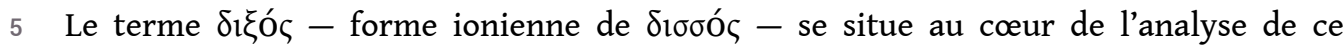
passage. En effet, les chercheurs ne s'accordent pas sur la signification à donner à l'adjectif dans ce contexte précis. Alors que certains estiment que l'expression $\delta_{1} \xi \grave{\alpha}$

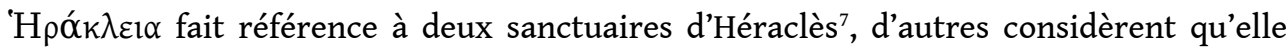
signifie « un sanctuaire d'Héraclès double »"

Chez Hérodote, $\delta$ ¡ óc est attesté 12 fois, dont 11 au pluriel. En raison du contexte dans lequel elle se situe, l'unique occurrence de $\delta 1 \xi o ́ \varsigma$ au singulier ne peut être traduite que 
par « double " " la notion de « deux » impliquant nécessairement un pluriel. Hérodote

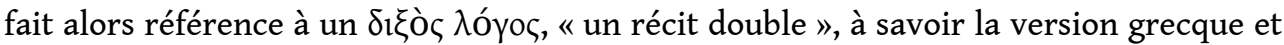
la version égyptienne de la mort de l'une des femmes de Cambyse ${ }^{10}$. Hérodote utilise la même expression au pluriel pour préciser que deux récits différents étaient racontés au sujet d'un certain Sophanès ${ }^{11}$. Ce cas est intéressant car il laisse penser que, lorsque

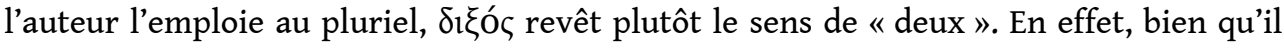

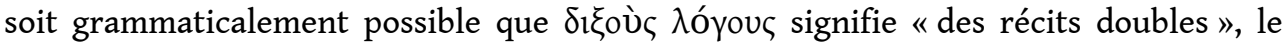
contexte du passage impose de traduire l'expression par "deux récits", la traduction "des récits doubles" suggérant que plusieurs récits présentaient deux versions. Les autres occurrences de l'adjectif semblent d'ailleurs confirmer que, au pluriel, $\delta_{1} \xi o ́ \varsigma$ signifie "deux » : il est en effet évident que Léonidas a deux frères aînés, Cléomène et Dorieus $^{12}$, et que les députés de Sparte affirment compatir aux malheurs des Athéniens parce que ces derniers ont été privés de deux récoltes, l'une l'été passé et l'autre l'été qui vient ${ }^{13}$. De même, Anaxandride vécut dans deux foyers ${ }^{14}$. Même évidence dans la description qu'Hérodote donne de la route menant de la mer d'Ionie à la demeure du Roi : aux frontières de la Cilicie, on franchit deux portes et deux postes de garde ${ }^{15}$. Pas de doute non plus concernant le prodige qui apparaît à Xerxès alors qu'il se trouve à Sardes: un poulain naît avec les parties intimes des deux sexes, à la fois mâles et femelles ${ }^{16}$. Par ailleurs, il est clair que l'adjectif $\delta_{1} \xi$ ó $\varsigma$ est employé à deux reprises pour distinguer deux parties d'un même ensemble : quand Hérodote précise qu'il existe des ibis de deux sortes ${ }^{17}$ et lorsqu'il affirme que, dans la même armée, sont regroupés deux types d'Éthiopiens ${ }^{18}$. Ce n'est que dans un seul passage que le sens de $\delta_{1} \xi_{\text {Kó }}$ s'avère moins évident : quand il décrit le monument d'Amasis qui se trouve dans la cour du

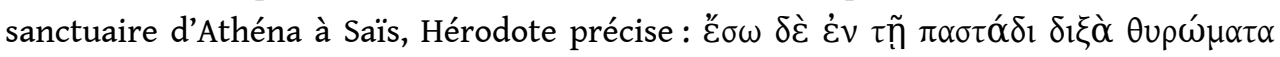

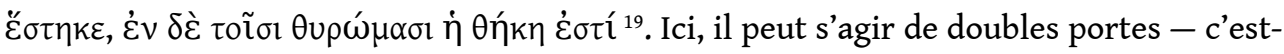
à-dire d'une porte à deux battants - ou de deux portes distinctes situées l'une derrière l'autre. Toutefois, selon A. Lloyd, la seconde interprétation serait probablement la bonne, en raison de l'existence de structures architecturales parallèles à Medinet $\mathrm{Habu}^{20}$.

7 Ainsi, lorsque $\delta_{1} \xi o ́ c$ se trouve au pluriel, il est apparemment toujours employé par Hérodote pour signifier "deux ». L'adjectif aurait-il donc la même signification que $\delta$ óo, au demeurant beaucoup plus fréquent dans l'œuvre d'Hérodote ${ }^{21}$ ? Ou bien, seraitil possible de distinguer une différence de sens, même minime, entre les deux termes ? Il convient pour ce faire de rechercher les cas où les substantifs qualifiés par $\delta_{1} \xi$ łó $\varsigma$ sont

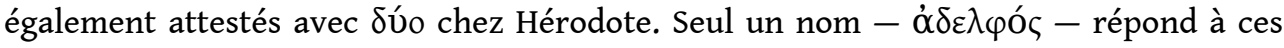
critères $^{22}$. Une analyse des différents contextes révèle qu'il n'y a a priori aucune raison

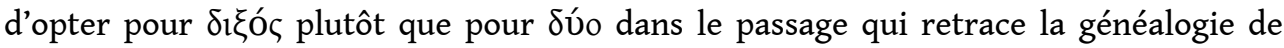
Léonidas. Il est donc vraisemblable que l'usage de l'un ou l'autre terme relève d'un souci de variatio de l'auteur. Une phrase à laquelle nous avons fait allusion auparavant semble d'ailleurs aller dans ce sens, puisqu'on y trouve à la fois $\delta$ v́o et $\delta$ iłóc sans

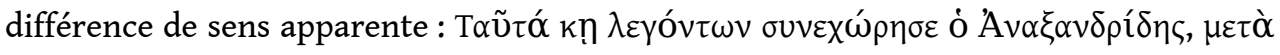

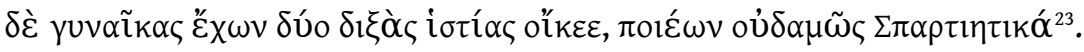

8 Afin d'approfondir cette analyse des significations de $\delta$ ı óc, il paraît nécessaire d'étendre la recherche des occurrences de l'adjectif aux auteurs athéniens du ve siècle

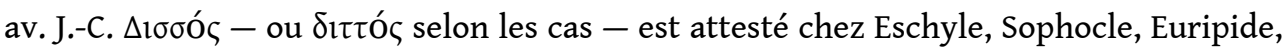
Aristophane, Antiphon, Isocrate, Platon et Xénophon. La plupart du temps, l'adjectif se trouve au pluriel et signifie «deux $»^{24}$. Il arrive d'ailleurs quelques fois, comme nous 


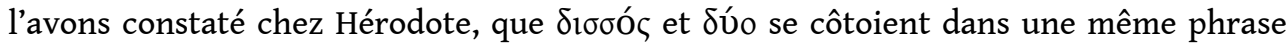
sans différence de sens perceptible ${ }^{25}$. Cependant, le sens de $\delta 1 \sigma \sigma o ́ \varsigma$ semble ajouter une dimension à celui de $\delta$ ó : l'adjectif qualifie fréquemment deux éléments - animés ou inanimés - intimement liés et formant parfois un tout indissociable. Ainsi en va-t-il par exemple des Atrides ${ }^{26}$, des Dioscures ${ }^{27}$, des membres d'une même famille ${ }^{28}$, des mains ${ }^{29}$ ou encore de la gymnastique et de la philosophie, deux disciplines complémentaires - l'une du corps et l'autre de l'âme ${ }^{30}$. La même notion d'unité pourrait également être perceptible dans un passage du Banquet de Xénophon où

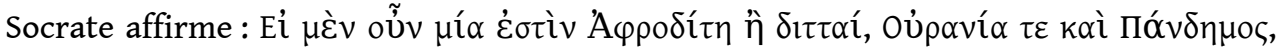
oủk oĩ $\delta \alpha^{31}$.

9 La différence entre $\delta ı \sigma o ́ \varsigma$ et $\delta$ v́o se révèle donc assez subtile : du fait qu'il est employé à plusieurs reprises pour qualifier deux éléments très proches, parfois presque

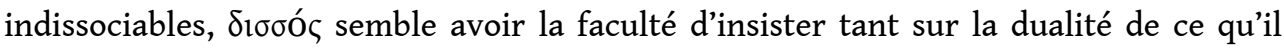
qualifie que sur une unité relative comme celle des mains, de frères ou de l'Aphrodite

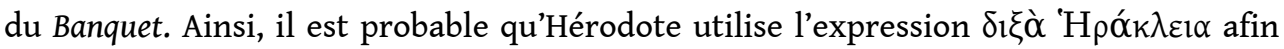
de mettre en évidence la dualité des lieux de cultes d'Héraclès tout en pointant vers l'unité de leur destinataire.

\section{'Enwvupín}

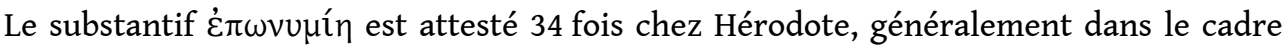
de l'explication de l'origine d'un nom de lieu, d'objet, de personne ou de groupe humain, au sens de "nom», "appellation ${ }^{32}$. Par ailleurs, sans compter le passage analysé, le terme s'applique à des dieux à cinq reprises, dont trois fois au sens de «nom », « qualificatif $~_{33}$. Les deux dernières occurrences de $\dot{\varepsilon} \pi \omega v v \mu i ́ n$ appliquées à des dieux s'inscrivent dans un contexte cultuel. Tout d'abord, au livre II, quelques lignes avant la conclusion du chapitre 44, Hérodote affirme qu'il a vu à Tyr un autre sanctuaire dédié à Héraclès sous le nom de Thasien ${ }^{34}$. Dans ce contexte, le substantif $\dot{\varepsilon} \pi \omega v \cup \mu i ́ n$ fait incontestablement référence au titre cultuel que porte le dieu. Il en est de même au livre VII, quand Hérodote explique que, depuis qu'ils ont adressé des prières et des vœux à Poséidon Sôter à la suite du naufrage des vaisseaux perses, les Grecs honorent Poséidon sous le nom - c'est-à-dire l'épiclèse - de Sôter ${ }^{35}$. Doit-on dès lors en déduire que l'expression 'O $\lambda \nu \mu \pi i ́ \omega$ غ̇ $\pi \omega v v \mu i ́ \eta v$, présente dans le passage qui nous occupe, signifie qu'Olympios est une épiclèse? Dans la perspective adoptée par Hérodote, il s'agit bel et bien d'un titre cultuel, puisqu'il est question de sacrifices:

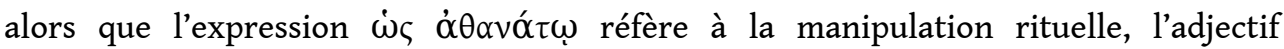

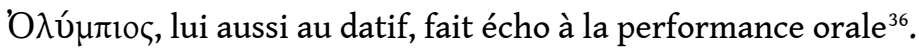

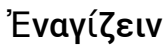

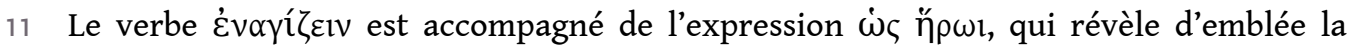
nature héroïque - et donc mortelle - du destinataire du sacrifice. La syntaxe de la

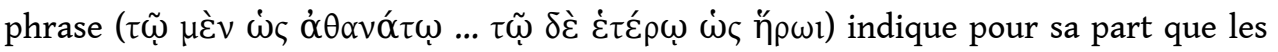
verbes $\dot{\varepsilon} v \alpha \gamma i ́ \zeta \varepsilon ı v$ et $\theta u ́ \varepsilon ı v$ se trouvent en opposition et font par conséquent allusion à deux types de rituels différents ${ }^{37}$. La seule autre attestation du verbe $\dot{\varepsilon} v \alpha \gamma i ́ \zeta \varepsilon v v$ chez Hérodote apparaît au livre $\mathrm{I}^{38}$, où le terme est employé pour désigner les sacrifices offerts aux prisonniers phocéens lapidés par les Agylléens ${ }^{39}$. Dans ce deuxième cas, le verbe s'applique donc également à des défunts, peut-être héroïsés eux aussi. 


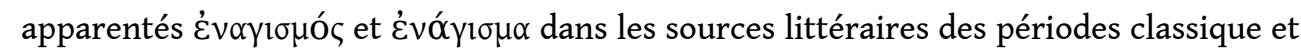
hellénistique concernent, elles aussi, uniquement des défunts et des héros ${ }^{40}$. Par ailleurs, bien que ces termes ne soient pas très précis à propos du contenu des rituels auxquels ils font référence, on y décèle en général une idée de soustraction de l'offrande à la consommation des sacrifiants ${ }^{41}$. Ainsi, غ̇vaүí indication tant sur le statut du destinataire du sacrifice que sur l'acte sacrificiel luimême ${ }^{42}$.

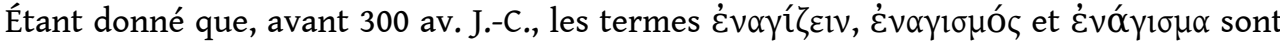
plus fréquemment utilisés dans le cadre de sacrifices offerts à des défunts ordinaires, G. Ekroth a émis l'hypothèse que la pratique de l'enagizein dans les cultes de héros fonctionnerait comme une manière de relier ces destinataires à la sphère mortelle. En effet, on observe chez eux un lien étroit avec la mort, perceptible par l'importance de leur tombe dans le culte, par la violence de leur mort ou encore par le contraste de leur culte avec celui d'un immortel ${ }^{43}$. Dans le passage d'Hérodote, cette opposition entre mortalité et immortalité concerne un même destinataire: la pratique de l'enagizein rappelle la mortalité originelle d'Héraclès, tandis que le sacrifice du type thysia indique que le héros a atteint le statut d'immortel ${ }^{44}$.

$\mathrm{Au}$ terme de cette analyse, la traduction la plus appropriée du passage étudié nous semble dès lors être la suivante :

Mes recherches montrent donc clairement qu'Héraclès est un dieu ancien; et ils me semblent agir de manière très sensée, ceux d'entre les Grecs qui, les ayant érigés, possèdent deux sanctuaires d'Héraclès, offrant à l'un des sacrifices comme à un immortel, Olympien quant à son surnom, tandis qu'à l'autre ils offrent des enagismata comme à un héros.

\section{La complexité d'Héraclès}

15 Après avoir mené des recherches en Égypte, en Phénicie et à Thasos, Hérodote parvient à la conclusion qu'Héraclès - qu'il rangeait $a$ priori au rang des immortels - est bien un dieu ancien, tant pour les Égyptiens que pour les autres peuples qu'il a rencontrés. Par ailleurs, il salue le bon sens de ceux qui, par des pratiques rituelles différentes, rendent hommage à la fois à un Héraclès-dieu et à un Héraclès-héros, en conservant fermement cette distinction. À la suite des travaux d'A. Verbanck-Piérard, nous pensons que, dans ce chapitre, Hérodote opère la rationalisation de deux données apparemment contradictoires : il tente de concilier l'existence d'un Héraclès-dieu, considéré comme tel par les Grecs dans leurs pratiques cultuelles, et celle d'un Héraclès-héros, mis en scène par l'épopée et les mythes. Pour ce faire, l'auteur en vient à distinguer deux Héraclès apparus à des périodes différentes de l'histoire et en des lieux distincts : le dieu, d'origine égyptienne, et le héros grec, fils d'Amphitryon et d'Alcmène ${ }^{45}$. Par conséquent, il est possible que la conclusion ne soit que la suite du raisonnement théorique de l'auteur et ait dès lors pour objectif de transmettre la recommandation suivante : bien qu'il soit évident qu'Héraclès est un dieu ancien, il est important, d'un point de vue cultuel, de ne pas négliger le héros Héraclès de l'épopée et des mythes. Ainsi, ces quelques lignes constitueraient peut-être davantage un exemple à suivre que le témoignage d'un double culte prenant place dans un ou des lieux définis ${ }^{46}$.

Bien que cette lecture soit en partie convaincante, la conclusion à laquelle A. VerbanckPiérard parvient dans son article de 1989 - la double nature d'Héraclès, à la fois divine 
et humaine, ne s'inscrirait en aucune manière dans la réalité cultuelle ${ }^{47}$ - mérite à nos yeux d'être nuancée. En effet, comme l'a déjà souligné E. Stafford, s'il est incontestable que les sources livrent surtout des exemples de cultes exclusivement divins d'Héraclès, certaines pratiques rituelles sont difficilement explicables sans référence à l'aspect héroïque du personnage ${ }^{48}$. Que l'on pense notamment à l'enateuein, mentionné dans un règlement rituel thasien qui fut, comme le texte d'Hérodote, à l'origine d'un grand nombre de commentaires et d'interprétations ${ }^{49}$.

\section{Le culte d'Héraclès à Thasos}

Étant donné que, dans sa réflexion sur Héraclès, Hérodote évoque un sanctuaire d'Héraclès Thasios à Tyr ainsi qu'un voyage à Thasos, de nombreux chercheurs ont considéré que, sous l'expression vague ỡ் Thasiens. Un tel raccourci n'est pas véritablement fondé: Hérodote n'affirme aucunement qu'il existait un double culte d'Héraclès à Thasos; il mentionne uniquement cette cité dans le cadre de son argumentation, afin de donner une preuve parmi d'autres de l'antiquité du dieu Héraclès. Cependant, il n'est pas impossible que l'auteur ait pensé aux Thasiens. Il semble dès lors intéressant d'étudier le culte d'Héraclès à Thasos - attesté tant par des sources épigraphiques qu'archéologiques afin de vérifier si ces Grecs qui possèdent deux sanctuaires d'Héraclès et pratiquent des rituels différents en son honneur peuvent effectivement être les Thasiens.

\section{L'Héracleion de Thasos}

Les fouilles entreprises à l'Héracleion de Thasos ont permis d'identifier la majeure partie des monuments qui se trouvaient dans le sanctuaire. Autour d'une esplanade centrale, sur laquelle subsistent les fondations d'un autel, s'élevaient plusieurs bâtiments : au nord un temple, à l'est une longue galerie - leschè - et au sud un édifice composé de cinq chambres renfermant 17 lits et s'ouvrant sur un portique (édifice aux oikoi). Le temple, construit au début du ve siècle av. J.-C., sous lequel les chercheurs ont retrouvé les vestiges d'un édifice plus ancien (daté entre la fin du viI siècle et le troisième quart $\mathrm{du} \mathrm{vl}^{\mathrm{e}}$ siècle av. J.-C.), fut entouré à l'époque hellénistique par une galerie composée de colonnes ioniques. La leschè, élevée au ve siècle av. J.-C., fut en partie reconstruite à l'époque hellénistique. Sur un bloc qui appartenait à sa façade ${ }^{50}$, les chercheurs ont mis au jour une inscription datant de la fin du $\mathrm{IV}^{\mathrm{e}}$ siècle ou du début $\mathrm{du} \mathrm{III}^{\mathrm{e}}$ siècle av. J.-C. et contenant le bail locatif d'un jardin d'Héraclès qui dépendait probablement du sanctuaire ${ }^{51}$. Lors de sa construction au ve siècle av. J.-C., l'édifice aux oikoi a englobé un bâtiment datant du troisième quart du vi siècle av. J.-C. - l'édifice en appareil polygonal - qui avait déjà pour fonction d'accueillir les participants aux banquets sacrificiels, et au centre duquel était installé un foyer. Derrière les chambres (oikoi), se trouvait une cour triangulaire dans laquelle était creusé un puits ${ }^{52}$. Des fouilles plus récentes entreprises dans le sanctuaire ont mis au jour des ossements animaux portant des traces de découpe ${ }^{53}$, qui, comme les oikoi, prouvent le déroulement de rituels participatifs. 


\section{Le règlement du culte d'Héraclès Thasios}

Une autre inscription qui atteste le culte d'Héraclès à Thasos fut mise au jour près du Passage des Théores, au nord-est de l'agora. Elle est datée du début du ve siècle av. J.-C. et contient les recommandations suivantes :

\begin{tabular}{|c|c|}
\hline & {$\left[{ }^{\prime} H \rho \alpha\right] \kappa \lambda \varepsilon \tilde{\imath} \Theta \alpha \sigma i ́ \omega \iota$} \\
\hline & 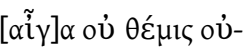 \\
\hline & 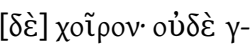 \\
\hline & 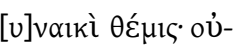 \\
\hline 5 & 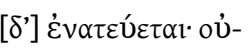 \\
\hline & 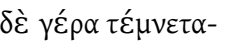 \\
\hline & 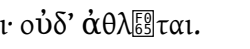 \\
\hline
\end{tabular}

À Héraclès Thasios, il n'est pas religieusement permis (de sacrifier) ni une chèvre ni un porcelet. Il n'est pas permis aux femmes (de participer). (5) Il n'est pas permis de faire une division en neuf parts. Aucune part d'honneur ne doit être découpée. Aucun concours n'a lieu ${ }^{54}$.

L'interdiction de sacrifier des chèvres et des porcelets est attestée dans plusieurs autres cultes, notamment à Thasos. Ainsi, de même que pour Héraclès Thasios, il n'est pas religieusement correct de sacrifier ces animaux à Peithô $\hat{o}^{55}$ ni aux Charites ${ }^{56}$. Par ailleurs, le porcelet est proscrit pour les Nymphes et Apollon Nymphagète ${ }^{57}$, tandis que la chèvre

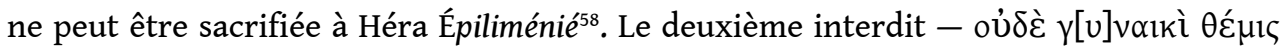
- ne trouve pas de parallèle à Thasos ${ }^{59}$, mais est attesté dans d'autres cités, tant dans des cultes d'Héraclès que d'autres divinités ${ }^{60}$. L'exclusion des femmes relève de dispositions exceptionnelles et spécifiques à certains cultes; elle a surtout pour but de délimiter l'identité d'un groupe et d'instaurer une intimité entre ce groupe et la divinité qu'il honore ${ }^{61}$.

Les trois dernières clauses s'avèrent plus problématiques. Outre sa présence dans ce

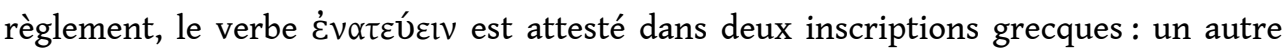
texte thasien ainsi qu'un calendrier sacrificiel de Myconos $^{62}$. L'inscription thasienne est

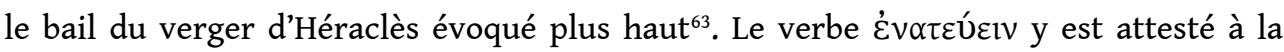
ligne 10 sous la forme $[-\dot{\varepsilon}] v \alpha \tau \varepsilon v \theta \tilde{\eta} 1$, précédé d'une lacune importante. L'inscription de Myconos est un calendrier cultuel presque intégralement conservé jusqu'à la ligne 40 et comportant peu de restitutions ${ }^{64}$. Aux lignes 23-24, qui réglementent le culte de

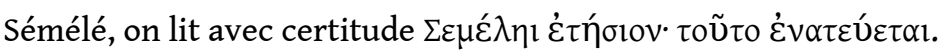

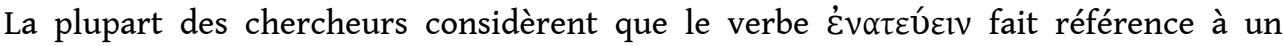
sacrifice dans lequel la part consumée est plus importante que la timè habituelle des dieux - ici, un neuvième de l'animal - mais où la majorité des parts consommables revient aux sacrifiants. Il s'agit de ce que S. Scullion appelle un moirocauste $e^{65}$. À l'époque où seul le texte de Myconos était connu, P. Stengel avait déjà émis cette hypothèse ${ }^{66}$, qui semble confirmée depuis la découverte de la fameuse inscription sacrificielle de Sélinonte datant de la première moitié du ve siècle av. J.-C. ${ }^{67}$. Aux lignes 11-12, le règlement prescrit pour les Tritopatreis «impurs" un sacrifice qui consiste en une 
division de l'animal en neuf parts et en la combustion complète de l'une d'entre elles

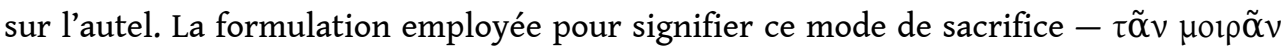

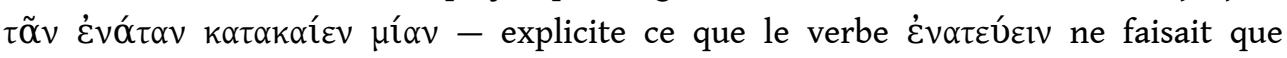
suggérer à propos du traitement de la neuvième part ${ }^{68}$.

Or, la pratique de l'enateuein étant proscrite dans le culte d'Héraclès Thasios, quel était le rituel à accomplir? Deux hypothèses peuvent être faites pour expliquer cette interdiction, associée à celles de découper des gèra - unique dans le corpus des normes rituelles ${ }^{69}$ - et d'organiser des $a t h l a^{70}$. La première hypothèse revient à considérer que la totalité de l'animal était soumise au feu, et pas seulement la neuvième part ${ }^{71}$. Cependant, même si l'argument n'est pas définitif, on s'attendrait davantage à l'emploi

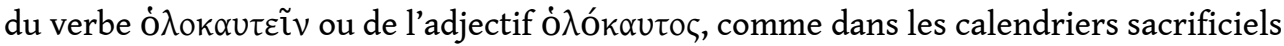
$d^{\prime}$ 'Erchia et de Thorikos ${ }^{72}$. La deuxième hypothèse consiste à voir dans le rituel prescrit une thysia ${ }^{73}$, d'autant plus que l'holocauste était une pratique sacrificielle marginale dans le monde grec ${ }^{74}$. Mais comment expliquer alors l'absence de gèra et d'athla ? Selon B. Bergquist, l'inscription réglementerait un culte privé d'Héraclès : l'absence de prêtre justifierait l'absence de parts d'honneur et le caractère privé du culte expliquerait également qu'on n'y tenait pas de concours, trop coûteux pour de simples particuliers ${ }^{75}$. Bien que ce raisonnement ait séduit certains chercheurs ${ }^{76}$, il n'est pas entièrement convaincant. En effet, il paraîtrait étrange que l'Héraclès Thasios - littéralement l'Héraclès de Thasos, donc une figure d'autant plus civique qu'elle porte le nom de la cité dans son épiclèse - soit honoré dans le cadre d'un culte privé ${ }^{77}$.

Par ailleurs, B. Bergquist considère que le règlement du culte d'Héraclès Thasios n'a pas été découvert in situ : bien qu'il ait été mis au jour près de l'agora, il réglementerait un culte qui se déroulait à l'Héracleion. Or, il n'y a aucune raison d'imaginer un quelconque déplacement de la pierre ou de supposer que l'inscription trouvée près du Passage des Théores fût une copie de l'originale présente à l'Héracleion ${ }^{78}$. Au contraire, plusieurs éléments laissent penser que le culte était pratiqué non loin du lieu de découverte de l'inscription, c'est-à-dire près de l'agora ou sur l'agora elle-même. Tout d'abord, les fouilles ont mis au jour un pied de table à offrandes portant les attributs d'Héraclès - la massue et la leontè - dans le même secteur ${ }^{79}$. Par ailleurs, à Thasos, l'épiclèse Thasios est attribuée à une seule autre divinité : Zeus Agoraios Thasios, dont le culte se tenait sur l'agora ${ }^{80}$.

Nous pensons donc qu'il existait à Thasos, outre l'Héracleion, un deuxième lieu de culte en l'honneur d'Héraclès, où ce dernier recevait probablement des thysiai sans parts d'honneur et où l'on n'organisait pas de concours ${ }^{81}$. Il est possible que l'interdiction de couper des gèra ait eu pour but de réserver plus de viande aux sacrifiants - en l'occurrence un groupe exclusivement masculin, dont la parité était ainsi tout particulièrement soulignée. Les clauses interdisant l'enateuein et la participation des femmes pourraient d'ailleurs avoir eu la même finalitée ${ }^{82}$. Quant à l'interdiction d'organiser des concours, étant donné que le culte se déroulait sur l'agora, on peut supposer qu'elle était formulée pour des raisons pratiques ${ }^{83}$.

\section{Le bail du verger d'Héraclès}

26 L'interdiction de l'enateuein dans le culte de l'agora laisse supposer que cette pratique était d'application dans un autre culte rendu à Héraclès dans la cité. Comme on l'a vu

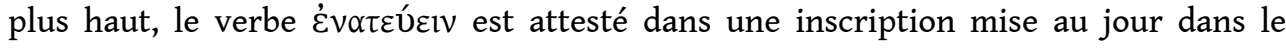


secteur de l'Héracleion - le bail du verger d'Héraclès. Une lacune malencontreuse juste avant la forme verbale empêche toutefois d'être assuré que l'enateuein ne faisait pas l'objet d'une interdiction là aussi ${ }^{84}$. Cependant, à partir du moment où l'on s'attend à trouver hors de l'agora un rituel où l'enateuein est permis, on peut faire l'hypothèse que c'est à l'Héracleion que ce sacrifice de la neuvième part avait lieu et que, dès lors, aucune négation n'affectait le verbe dans la lacune de l'inscription du bail ${ }^{85}$. Quant à l'archéologie de l'Héracleion, elle ne contredit pas cette hypothèse : la présence d'oikoi pourvues de lits ainsi que la découverte d'ossements portant des traces de découpe indiquent assurément une consommation des viandes qui, dans le cas d'un enateuein, s'applique à huit neuvièmes de l'animal. Cependant, les restes fauniques révèlent également des prélèvements des fémurs et d'autres parties des pattes en vue d'une combustion sur l'autel ${ }^{86}$, ce qui semble suggérer l'accomplissement d'un type de thysia dans ce sanctuaire. L'enateuein pourrait alors avoir été facultatif dans le culte de l'Héracleion, tandis qu'il était interdit dans celui de l'agora.

\section{Héraclès : dieu ou héros ?}

27 Jusqu'à présent, nous avons uniquement abordé les rituels accomplis pour Héraclès, laissant volontairement de côté la question de la nature du destinataire. En effet, il nous semblait primordial d'examiner les sources thasiennes sans chercher à vérifier directement si les Thasiens honoraient Héraclès à la fois comme un dieu et comme un héros. À l'heure actuelle, on a renoncé à décrire les sacrifices grecs selon une stricte polarité entre rituels olympiens et rituels chthoniens. Les données disponibles sont bien plus complexes et subtiles, mettant en évidence divers marqueurs rituels signalant l'identité immortelle ou mortelle des destinataires ${ }^{87}$. Par ailleurs, il est désormais acquis qu'il n'y avait pas de différence nette entre les rites sacrificiels divins et héroïques, et que la majorité des sacrifices offerts aux héros étaient des thysiai, tout comme pour les dieux ${ }^{88}$.

La présence d'une thysia ne suffit donc pas à déterminer la nature divine d'un destinataire. Cependant, l'existence d'un certain nombre de cultes divins en l'honneur d'Héraclès dans le monde grec ${ }^{89}$ autorise à croire que, à Thasos également, Héraclès était considéré comme un dieu. Le fait qu'il porte l'épiclèse Thasios dans le culte de l'agora laisse d'ailleurs penser qu'il s'agit de l'olympien dont Hérodote parle, et donc le dieu dans sa pleine identité d'immortel, honoré par les "mâles» de la cité. En revanche, bien que les moirocaustes ne soient pas l'apanage des héros, l'enateuein semble être un rituel particulièrement héroïque ${ }^{90}$, pratiqué en l'honneur de figures au statut ambigu. En effet, si l'on met en regard les deux autres destinataires pour lesquels cette pratique est attestée - Sémélé à Myconos et les Tritopatreis «impurs » à Sélinonte -, on remarque qu'il s'agit de figures mortelles. En outre, le parallèle entre Héraclès et Sémélé est frappant: nés mortels, ils ont tous deux atteint l'immortalité grâce à l'apothéose, après avoir connu une mort violente où le feu joue un rôle primordial. Tandis qu'Héraclès est brûlé sur un bûcher érigé sur le mont đta, Sémélé est foudroyée à la vue de Zeus. Ainsi, bien qu'Héraclès soit incontestablement un destinataire divin aux yeux des Grecs, il semble que, dans une cité comme Thasos, on rendait hommage à la part mortelle du dieu par le biais d'une manipulation particulière de la viande ${ }^{91}$. 


\section{Conclusion} où se tenait une thysia égalitaire entre les hommes de la cité, et l'Héracleion, où étaient peut-être accomplis tant un enateuein - faisant place au statut mortel d'Héraclès qu'une thysia - reconnaissant un statut d'immortel au fils de Zeus.

Dès lors, s'il est évident que le passage d'Hérodote ne reflète pas l'ensemble des pratiques sacrificielles en l'honneur d'Héraclès dans le monde grec, il est en revanche tout à fait possible que l'auteur ait fait référence à la situation de Thasos, où deux sanctuaires et - si le point de vue développé plus haut est valide - deux types de rituels font écho à la figure complexe de celui que Pindare nomme ň $\rho \omega \varsigma$ - $\theta \varepsilon o ́ c$.

\section{Abréviations}

\begin{tabular}{|l|l|}
\hline Bailly & $\begin{array}{l}\text { A. BAILLY, Dictionnaire grec-français, rédigé avec le concours de E. EGGER, édition revue par } \\
\text { L. SÉCHAN, P. CHANTRAINE, Paris, 2000 [1894]. }\end{array}$ \\
\hline CGRN & $\begin{array}{l}\text { J.-M. CARBON, S. PEELS, V. PIRENNE-DELFORGE, A Collection of Greek Ritual Norms (CGRN), Liège 2016- } \\
\text { (http://cgrn.ulg.ac.be, consulté en 2016). }\end{array}$ \\
\hline LSCG & F. SOKOLOWSKI, Lois sacrées des cités grecques, Paris, 1969. \\
\hline LSJ & H.G. LIDDELL, R. SCOTT, H.S. JONES, A Greek-English Lexicon, $9^{\text {th }}$ ed., Oxford, 1961. \\
\hline LSS & F. SOKOLOWSKI, Lois sacrées des cités grecques. Supplément, Paris, 1962. \\
\hline NGSL & $\begin{array}{l}\text { E. LUPU, Greek Sacred Law. A collection of New Documents, Leiden, 2005 (Religions in the Graeco- } \\
\text { Roman World, 152). }\end{array}$ \\
\hline
\end{tabular}




\section{BIBLIOGRAPHIE}

D. ASHERI, A.B. LLOYD, A. CORCELLA, A Commentary on Herodotus Books I-IV, édité par O. MURRAY,

A. MOREno, Oxford, 2007.

B. BERGQUIST, Herakles on Thasos, Uppsala, 1973.

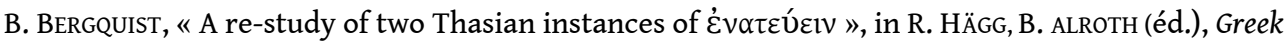
Sacrificial Ritual, Olympian and Chthonian. Proceedings of the Sixth International Seminar on Ancient Greek Cult, Stockholm, 2005, p. 61-70.

C. BONNET, Melqart. Cultes et mythes de l'Héraclès Tyrien en Méditerranée, Louvain/Namur, 1988 (Studia Phoenicia, 8).

J. CASABONA, Recherches sur le vocabulaire des sacrifices en grec des origines à la fin de l'époque classique, Aix-en-Provence, 1966.

S.G. COLE, « Gynaiki ou themis: Gender difference in the Greek leges sacrae », Helios 19 (1992), p. 104122 .

J. DES COURTILS, A. GARDEISEN, A. PARIENTE, « Sacrifices d'animaux à l'Hérakleion de Thasos », BCH 120 (1996), p. 799-820.

G. ЕкRотн, The Sacrificial Rituals of Greek Hero-Cults, Liège, 2002 (Kernos, suppl. 12).

G. ЕКRоTH, « Burnt, cooked or raw? Divine and human culinary desires at Greek animal sacrifice », in E. Stavrianopoulou, A. Michaels, C. Ambos (éd.), Transformations in Sacrificial Practices From Antiquity to Modern Times. Proceedings of an International Colloquium, Heidelberg 12-14 July 2006, Berlin, 2008, p. 87-112.

S. GEORGOUDI, « Héraclès dans les pratiques sacrificielles des cités », in C. BONNET, C. JOURDAINANNEQUIN, V. PIRENNE-DELFORGE (éd.), Le Bestiaire d'Héraclès : III Rencontre héracléenne, Liège, 1998 (Kernos, suppl. 7), p. 301-317.

S. GEORGoudi, « Réflexions sur des sacrifices et des purifications dans la 'loi sacrée' de Sélinonte », in A. IANNUCCI, F. MUCCIOLI, M. ZACCARINI (a cura di), La città inquieta. Selinunte tra lex sacra $e$ defixiones, Milan, 2015, p. 205-240.

Y. GRANDJEAN, F. SAlviat, Guide de Thasos, Paris, 2000.

T. HARRISON, Divinity and History. The Religion of Herodotus, Oxford, 2000.

M.H. JAMESON, D.R. JORDAN, R.D. KOTANSKI, A 'lex sacra' from Selinous, Durham/North Carolina, 1993

(GRBS Monographs, 11).

C. JOURDAIN-ANNEQUIN, « Public ou privé ? À propos de quelques cultes d'Héraclès dans la cité grecque ", Ktèma 23 (1998), p. 345-364.

M. LAUNEY, « Une dédicace thasienne à Héraklès », BCH 58 (1934), p. 173-183.

M. LAUNEY, «Inscriptions de Thasos », BCH 58 (1934), p. 484-500.

M. LAUNEY, « Le verger d'Héraclès à Thasos », BCH 61 (1937), p. 380-409.

P. LÉVÊQUE, A. VERBANCK-PIÉRARD, « Héraclès héros ou dieu ? », in C. BONNET, C. JOURDAIN-ANNEQUIN (éd.), Héraclès : d'une rive à l'autre de la Méditerranée. Bilan et perspectives, Bruxelles/Rome, 1992, p. 43-65. 
A.B. LLOYD, Herodotus Book II. Commentary 1-98, Leiden, 1976 (Études préliminaires aux religions orientales dans l'empire romain, 43).

A.B. LLOYD, Herodotus Book II. Commentary 99-182, Leiden, 1988 (Études préliminaires aux religions orientales dans l'empire romain, 43).

R. PARKER, « $\dot{\omega} \varsigma$ ท̆ $\rho \omega \mathrm{l} \dot{\varepsilon} v \alpha \gamma i ́ \zeta \varepsilon \mathrm{ElV}$ », in R. HÄGG, B. ALROTH (éd.), Greek Sacrificial Ritual, Olympian and Chthonian. Proceedings of the Sixth International Seminar on Ancient Greek Cult, Stockholm, 2005, p. 3745.

S. Peels, Hosios: A Semantic Study of Greek Piety, Leiden, 2016 (Mnemosyne, suppl. 387).

V. PIRENNE-DELFORGE, «Les rites sacrificiels dans la Périégèse de Pausanias », in D. KNOEPFLER,

M. PIÉRART (éd.), Éditer, traduire, commenter Pausanias en l'an 2000. Actes du colloque de Neuchâtel et de Fribourg (18-22 septembre 1998), Genève, 2001, p. 109-134.

V. PIRENNE-DELFORGE, Retour à la source. Pausanias et la religion grecque, Liège, 2008 (Kernos, suppl. 20).

V. PIRENNE-Delforge, G. Pironti, « Many vs. One », in E. Eidinow, J. Kindt (éd.), The Oxford Handbook of Ancient Greek Religion, Oxford, 2015, p. 39-47.

J. Pouilloux, Recherches sur l'histoire et les cultes de Thasos. De la fondation de la cité à 196 av. J.-C., Paris, 1954 (Études Thasiennes, 3).

J.E. PowELL, A Lexicon to Herodotus, Hildesheim, 1960.

G. Roux, «L'Hérakleion thasien. Problèmes de chronologie et d'architecture », Thasiaca, BCH Suppl. V (1979), p. 191-211.

J. RUDHARDT, Notions fondamentales de la pensée religieuse et actes constitutifs du culte dans la Grèce classique, Paris, $1992^{2}$ [1958].

F. SALVIAT, « Une nouvelle loi thasienne : institutions judiciaires et fêtes religieuses à la fin du IV s. av. J.-C. », BCH 82 (1958), p. 193-267.

S. SCULLION, « Heroic and Chthonian Sacrifice: New Evidence from Selinous », ZPE 132 (2000), p. $163-171$

S. SCULLION, « Sacrificial Norms, Greek and Semitic: Holocausts and Hides in a Sacred Law of Aixone ", in P. BRULÉ (éd.), La norme en matière religieuse en Grèce ancienne, Liège, 2009 (Kernos, suppl. 21), p. 153-169.

H. SEYRIG, « Quatre cultes de Thasos », BCH 51 (1927), p. 178-233.

E. STAFFORD, « Héraklès : encore et toujours le problème du heros-theos ", Kernos 18 (2005), p. 391406.

P. STENGEL, Opferbraüche der Griechen, Leipzig/Berlin, 1910.

D. VAN BERCHEM, « Sanctuaires d'Hercule-Melqart. Contribution à l'étude de l'expansion phénicienne en Méditerranée », Syria 44 (1967), p. 73-109.

F.T. VAN STRATEN, Hierà kalá. Images of Animal Sacrifice in Archaic and Classical Greece, Leiden, 1995 (Religions in the Graeco-Roman World, 127).

A. VERBANCK-PIÉRARD, « Le double culte d'Héraklès : légende ou réalité ? », in A.-F. LAURENS (éd.), Entre hommes et dieux. Le convive, le héros, le prophète, Besançon/Paris, 1989, p. 43-65. 


\section{NOTES}

1. .Hérodote, II, 42.

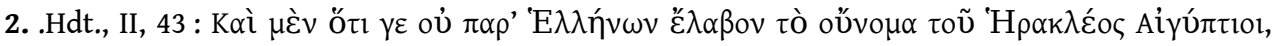

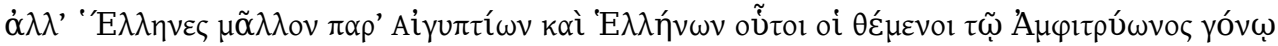

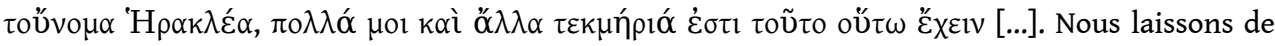

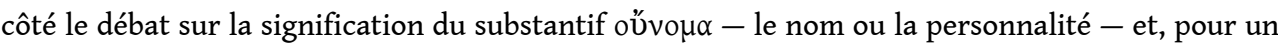
aperçu de la problématique, nous renvoyons à LLOYD (1976), p. 203-205 ; HARRISON (2000), p. 251264 ; ASHERI - LLOYD - CORCELLA (2007), p. 269.

3. Sur les différents types d'arguments, voir ASHERI - LLOYD - CORCELLA (2007), p. 229-230 et 269.

4. .Iepóv : Hdt., I, 105, 3 ; 143, 3 ; 172, 2 ; II, 41, 5 ; 42, 1 ; 44, 2 ; 44, 3 ; 44, 4 ; 56, 2 ; 99, 4 ; 155, 1 ; 182, 2 ; III, 142, 4 ; IV, 62, 1 ; 103, 2 ; 149, 2 ; V, 61, 2 ; VI, 105, 3 ; VII, 189, 3 ; 200, 2 ; IX, 97 ; پ̌ $\gamma \alpha \lambda \mu \alpha$ :

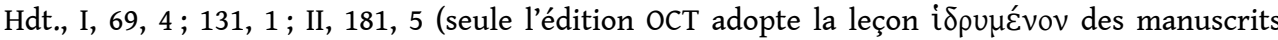

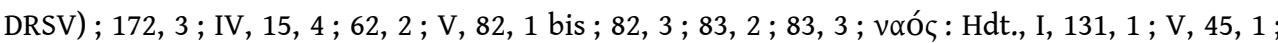

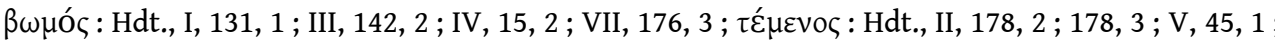

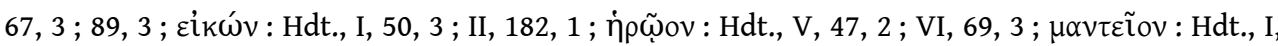
157, 3 ; II, 54, 1. Bien entendu, dans le cas d'un oracle, comme dans celui d'un sanctuaire, la fondation n'implique pas nécessairement la construction d'une structure matérielle. Il

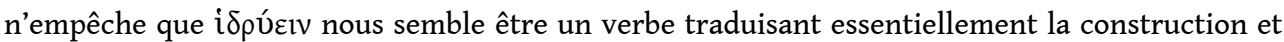
la matérialité.

5. .Dans quatre autres cas, le nom qui s'y rapporte est une cité et le verbe a le sens de "fonder", « établir » : Hdt., I, 142, 1 ; 142, 4 ; II, 59, 2 ; VIII, 120. On relève six cas où le verbe est associé au

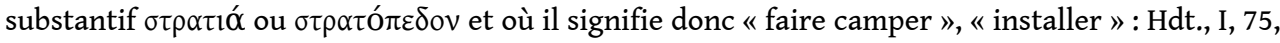
5 ; II, 118, 2 ; IV , 124, 1; 203, 3 ; VII, 127, 1; IX, 57, 2. Dans un seul cas, le verbe s'applique à un

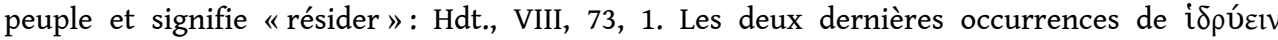
présentent un sens davantage métaphorique, «établir », en parlant de puissance, et «vivre » Hdt., II, 169, 2 ; VI, 86, a 4.

6. .Le substantif ǐ $\delta \rho \mu \alpha$ est quant à lui attesté une seule fois chez Hérodote (VIII, 144, 2) et

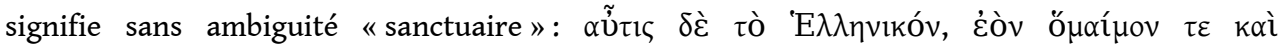

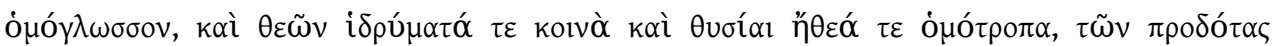

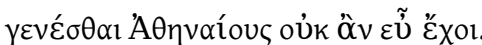

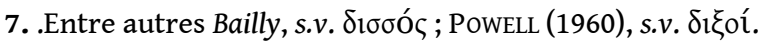

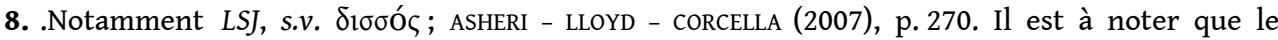
raisonnement de Lloyd est exclusivement fondé sur l'opinion - désormais contestée - qu'il existait des preuves architecturales d'un double culte, à la fois divin et héroïque, à l'Héracleion de Thasos. Nous y reviendrons plus loin. Avant toute chose, nous voudrions relever une

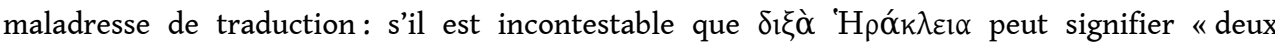
sanctuaires d'Héraclès ", il nous paraît imprécis de traduire cette expression au pluriel par le singulier « un sanctuaire d'Héraclès double ». En effet, s'il s'avérait que $\delta_{1} \xi o ́ c$ revêtait bien le sens de « double » dans ce contexte, il serait plus satisfaisant de traduire « des sanctuaires d'Héraclès doubles ».

9. .Dans un autre contexte, l'adjectif peut signifier "divisé » ou «à double sens ", « équivoque ». Voir par exemple Eschyle, Agamemnon, 122 ; Sophocle, Électre, 645.

10. .Hdt., III, 32, 2.

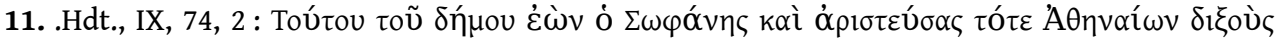

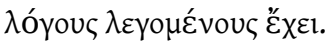

12. .Hdt., VII, 205, 1.

13. .Hdt., VIII, 142, 3.

14. .Hdt., V, 40, 2 
15. .Hdt., V, 52, 2 bis.

16. .Hdt., VII, 57, 2.

17. .Hdt., II, 76, 1.

18. .Hdt., VII, 70, 1.

19. .Hdt., II, 169, 5 .

20. .LLOYD (1988), p. 205 ; ASHERI - LLOYD - CORCELLA (2007), p. 368.

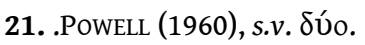

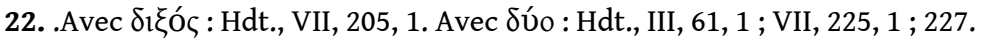

23. .Hdt., V, 40, 2 : «Ceux-ci tenant à peu près ces paroles, Anaxandride consentit et ainsi, ayant deux femmes, il habita deux foyers, ne faisant aucun cas des usages de Sparte ».

24. .Voir, par exemple, Soph., Philoctète, 264 ; Euripide, Hélène, 8 ; Platon, Lois, 932 e 4 ; Critias, 113 e 3 ; Xénophon, Art de la chasse, 3, 1, 1. Chez Soph., Électre, 645, l'adjectif au pluriel revêt le

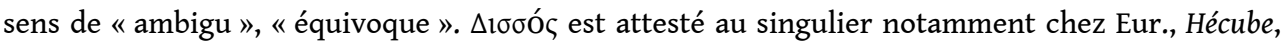
897 ; Plat., Théétète, 198 d 2 ; Gorgias, 517 c 8.

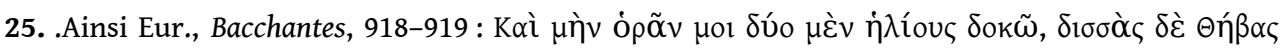

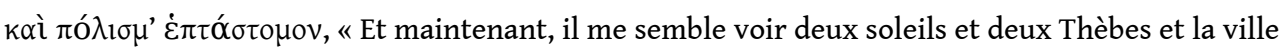

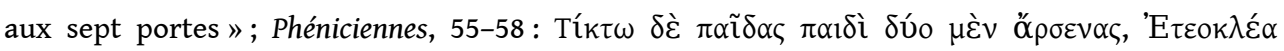

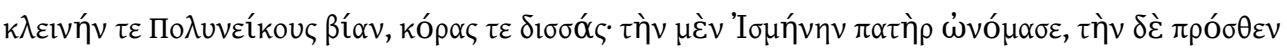

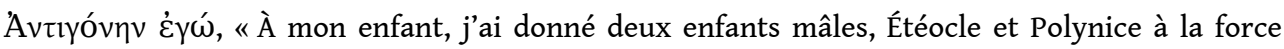
glorieuse, et deux filles. L'une, son père l'a appelée Ismène; l'autre, l'aînée, je l'ai appelée

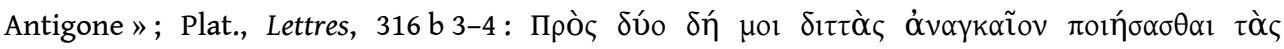
$\dot{\alpha} \pi 0 \lambda$ oүí $\alpha_{\varsigma}[. .$.$] , « Contre deux accusations, il m’est nécessaire de faire deux défenses [...] ».$

26. .Notamment Esch., Agamemnon, 122 ; Soph., Ajax, 57 ; 947 ; Eur., Hécube, 510 ; Oreste, 818.

27. .Eur., Hélène, 1643 ; Iphigénie à Aulis, 768.

28. .Par exemple Soph., Antigone, 971 ; Eur., Hécube, 1051 ; Médée, 1395 ; Phéniciennes, 427 ; 1243.

29. .Eur., Troyennes, 1306.

30. .Plat., Lois, $795 \mathrm{~d} 6$; Isocrate, $\mathrm{XV}, 181,4$.

31. .Xén., Banquet, 8, 9, 2. Cet exemple est toutefois ambigu car l'interprétation de l'adjectif

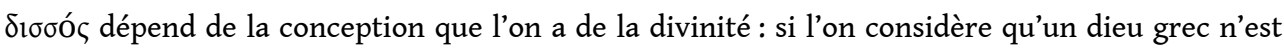
pas atomisé en fonction de ses épiclèses, on verra dans l'adjectif le renvoi à l'unité de la figure divine. Si l'on considère au contraire que le dieu se « divise » en autant d'épiclèses qu'il porte, on

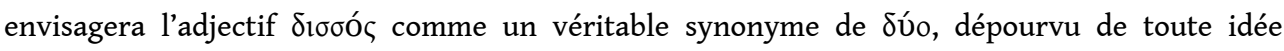
d'unité. Sur cette problématique, voir PIRENNE-DELFORGE - PIRONTI (2015).

32. .Lieu : Hdt., IV, 45, 2 bis ; 45, 3 ; 148, 4 ; VII, 58, 3 ; 121, 1 ; 178, 2. Objet : Hdt., I, 14, 3. Personne : Hdt., IV, 155, 2 ; V, 65, 4 ; 92 e 1. Groupe humain : Hdt., I, 94, 7 ; 173, 3 ; II, 42, 5 ; IV , 6, 2 ; 107 ; V, 68,$1 ; 68,2$; VII, 61, 3;74, $1 ; 91$; 92. Dans six autres cas, le sens est identique, bien que le contexte ne soit pas celui de l'explication de l'origine d'un nom : Hdt., II, 17, 2 ; IV, 15, 2 ; 15, 4 ; V, 66,2 bis ; VI, 53, 2.

33. .Hdt., II, 4,$2 ; 52,1 ; 53,2$.

34. .Hdt., II, 44, 3.

35. .Hdt., VII, 192, 2.

36. Si le dieu Héraclès est appelé Olympios, le héros n'est quant à lui pas assorti d'un surnom dans la construction du passage d'Hérodote.

37. .Comme l'a montré CASABONA (1966), p. 84-85, 204 et 337, la relation entre le verbe $\dot{\varepsilon} v \alpha \gamma i ́ \zeta \varepsilon ı v$ et le verbe $\theta$ úcıv est celle d'un terme technique, marqué - sacrifier à un mort, héroïsé ou non -,

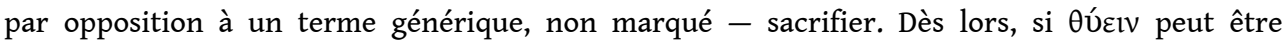
employé pour signifier des sacrifices de tous types, offerts tant à des dieux qu'à des héros ou des

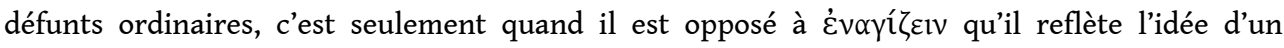
sacrifice à un immortel. 
38. .Hdt., I, 167, 2.

39. .Le lieu de la lapidation étant devenu un endroit dangereux, où tout être originaire d'Agylla qui y passait se retrouvait estropié ou paralysé, les Agylléens consultèrent la Pythie, qui leur ordonna de sacrifier aux défunts Phocéens et d'organiser en leur honneur des jeux gymniques et équestres.

40. .Nous renvoyons à EKROTH (2002), p. 82 et 87 pour les tableaux récapitulatifs des occurrences de ces différents termes.

41. RUDHARDT (1992²), p. 239.

42. .Cf. PiRENNE-DELFORGE (2000), p. 133, qui constate d'ailleurs (p. 122), en mettant en regard les

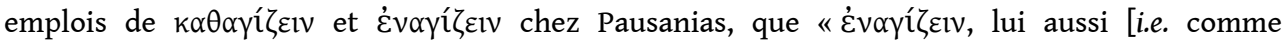
$\kappa \alpha \theta \alpha \gamma i ́ \zeta \varepsilon v v]$ chargé de l'idée de destruction, oriente davantage la pratique vers l'identité du destinataire du sacrifice, dont on expose ainsi la face héroïque. »

43. .ЕКRоTH (2002), p. 88-89.

44. .ЕКRоTн (2002), p. 126-127.

45. .VERBANCK-PIÉRARD (1989), p. 46.

46. .VERBANCK-PIÉRARD (1989), p. 47.

47. .VERBANCK-PIÉRARD (1989), p. 54 : « En second lieu, l'Apothéose ne s'inscrit nullement dans les rites. Le culte ne dit pas la double nature d'Héraclès. " Avant les recherches entreprises par VERBANCK-PIÉRARD (1989), p. 43-65 ; LÉVÊQUE - VERBANCK-PIÉRARD (1992), p. 43-65 et GEORGOUdi (1998), p. 301-317, l'existence d'un double culte en l'honneur d'Héraclès - à la fois héroïque et divin -, qui traduirait l'ambiguïté mythique du personnage, était tenue pour assurée.

48. STAFFORD (2005), p. 392.

49. IG XII Suppl. 414 ; LSS 63 ; CGRN 27.

50. .LAUNEY (1937), p. 380.

51. IG XII Suppl. 353. Outre cette inscription, l'Héracleion a également livré deux dédicaces à Héraclès. Cf. LAUNEY (1934), p. 173-183 et 484-491. Un calendrier des fêtes (ca. 300 av. J.-C.), retrouvé sur l'agora, et un décret (ca. 350 av. J.-C.) témoignent par ailleurs de deux fêtes en l'honneur d'Héraclès : les Sôteria, pour Héraclès Sôter, et les Grandes Héracleia, où se déroulaient des concours athlétiques. Cf. SALViat (1958), p. 193-267 et Pouilloux (1954), nº 141.

52. .GRANDJEAN - SALVIAT (2000), p. 142-145. Avant les mises au point de BERGQUIST (1973) et de RouX (1979), p. 191-211, certains éléments architecturaux de l'Héracleion étaient considérés comme des preuves d'un double culte d'Héraclès, à la fois héroïque et divin. Tandis qu'on considérait le temple ionique comme un temple d'Héraclès-dieu, on pensait que l'édifice polygonal était un temple d'Héraclès-héros. En effet, le foyer et le puits étaient interprétés comme des structures de type héroïque et chthonien : une eschara d'une part et un bothros de l'autre. L'étude des termes eschara et bothros entreprise par Е КRОТн (2002), p. 25-74 confirme que ces éléments architecturaux ne peuvent être considérés comme héroïques.

53. DES COURTILS - GARDEISEN - PARIENTE (1996), p. 800.

54. .Traduction empruntée à CGRN 27.

55. .IG XII Suppl. 394 ; LSS 73 ; CGRN 178 (à paraître).

56. .IG XII 8, 358 (CGRN 17); LSCG 114 B.

57. IG XII 8, 358 (CGRN 17); LSCG $114 \mathrm{~A}$.

58. .IG XII Suppl. 409 ; LSS 74 ; CGRN 23.

59. .Dans le culte d'Athéna Patrôié, qui se tenait dans le Thesmophorion de Thasos, les femmes étaient au contraire expressément incluses. Cf. LSCG 113 ; CGRN 18.

60. .COLE (1992), p. 105, dresse une liste des cas d'exclusion de femmes dans les inscriptions.

61. .PeELS (2016), p. 204-205. 
62. Pouilloux mentionne une troisième attestation du verbe à Thasos. Cf. PouilLoux (1954), p. 82, $\mathrm{n}^{\circ} 10 \mathrm{a}$. Cependant, en raison du caractère extrêmement fragmentaire de l'inscription, nous n'en tiendrons pas compte.

63. .Nous renvoyons à LAUNEY (1937) pour une analyse approfondie de l'inscription.

64. .LSCG 96 (CGRN 156 [à paraître]).

65. .SCULLION (2000), p. 165-166.

66. .STENGEL (1910), p. 132.

67. .JAMESON - JORDAN - KOTANSKY (1993) ; NGSL 27 (CGRN 13). GEORGOUDI (2015) revient brièvement sur la pratique de l'enateuein dans le cadre d'une réflexion sur les sacrifices et les purifications attestés dans cette inscription.

68. .Depuis la découverte de l'inscription de Sélinonte, les interprétations de Sokolowski (commentaire de LSS 63) et BERGQUIST (1973), p. 70-80, sont dépassées. B ERGQUIST (2005) est d'ailleurs revenue sur l'hypothèse proposée dans sa monographie de 1973, selon laquelle le verbe serait relatif à une taxe.

69. .CGRN 27, commentaire de la 1. 6 .

70. Cette clause est généralement comprise comme une interdiction de prélever des parts sacrificielles en guise de prix pour les vainqueurs des concours. Or, au passif, le verbe $\dot{\alpha} \theta \lambda \varepsilon \dot{\varepsilon} \omega$ revêt le sens de "lutter dans des jeux", "organiser des concours». Cf. LSJ, s.v. $\dot{\alpha} \theta \lambda \varepsilon \dot{\varepsilon} \omega$. La cinquième clause - oủ $\delta^{\prime} \dot{\alpha} \theta \lambda$ 熙 $\tau \alpha 1$ - appartient donc probablement aux règles concernant la participation et le comportement dans le sanctuaire. Cf. CGRN 27, commentaire de la ligne 7.

71. SEYRIG (1927), p. 194-195, suivi notamment par LAUNEY (1937), p. 399 ; VAN BERCHEM (1967), p. 100 ; DES COURTILS - GARDEISEN - PARIENTE (1996), p. 800 ; SCULLION (2000), p. 166 ; STAFFORD (2005), p. 396-397. Selon BERGQUIST (1973), p. 71-72, cette hypothèse n'est pas valide car cela n'aurait aucun sens d'interdire de couper des gèra dans le cadre d'une pratique où la totalité de l'animal disparaît. Or SCULLION (2009) a montré que les peaux pouvaient être attribuées au prêtre lors d'un holocauste. Dès lors, l'interdiction de prélever des gèra serait tout à fait justifiée et aurait pour but de préciser que la totalité de l'animal était brûlée, y compris la peau qui aurait pu servir de part d'honneur.

72. Erchia: LSCG 18 ; Thorikos: NGSL 1. Cf. PEELS (2016), p. 199, n. 126.

73. .L'hypothèse de la thysia est notamment partagée par Sokolowski (LSS, p. 121); BERGQUIST (2005), p. 64-65 ; LUPU (2005), p. 373.

74. .ЕкRотн (2008), p. 89-90. Selon F.T. Van Straten, une œnochoé attique de la fin du ve siècle av. J.-C. attesterait un holocauste pour Héraclès. Cf. VAN STRATEN (1995), p. 157-158, fig. 168. Cette interprétation est cependant contestée par ЕКROTH (2002), p. 289-290, n. 377.

75. .BERGQUIST (2005), p. 62-65. L'hypothèse du culte privé est empruntée à VAN BERCHEM (1967), p. 95-96.

76. .Ainsi ЕКROTH (2002), p. 221, n. 29.

77. JOURDAIN-ANNEQUIN (1998), p. 352 : «On ne peut être plus "public" que cet Héraclès qui s'avoue "politique" au sens premier du terme."

78. .Pour BonNET (1988), p. 359, l'Héraclès Thasios « ne pouvait être que le grand dieu de Thasos, celui qui avait pour siège l'Héracleion monumental ». STAFFORD (2005), p. 397, pense elle aussi que l'inscription faisait référence à un culte accompli à l'Héracleion. En revanche, elle considère que le rituel prescrit était un holocauste et que le règlement reflétait une mesure extraordinaire, peut-être en lien avec la situation politique perturbée de l'époque.

79. DES COURTILS - GARDEISEN - PARIENTE (1996), p. 800.

80. .L'inscription IG XII 8, 361 a permis d'identifier le sanctuaire du dieu. Cf. GRANDJEAN - SALVIAT (2000), p. 76.

81. CGRN 27, commentaire de la 1.6: «A relatively 'normal' sacrifice for Heracles, but with no attribution of priestly portions, remains a distinct and likely possibility. » 
82. Sokolowski, cité par PeELS (2016), p. 204-205, n. 143.

83. .CGRN 27, commentaire de la 1. 7.

84. .BERGQUIST (2005), p. 68-69, postule la présence d'une négation dans la lacune. À ses yeux, l'inscription ferait allusion à un culte où l'on accomplissait une thysia «normale " - avec découpe de parts d'honneur -, et où se tenaient des concours. Il aurait donc existé deux cultes d'Héraclès célébrés à l'Héracleion et caractérisés par une thysia, mais différenciables par leur caractère tantôt privé, tantôt public.

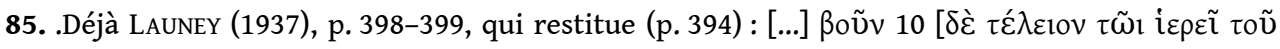

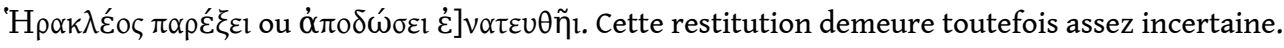
86. DES COURTILS - GARDEISEN - PARIENTE (1996), p. 804-805.

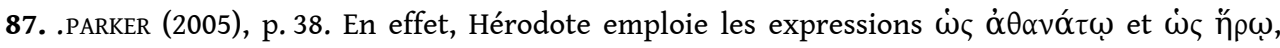
opérant une distinction entre immortel et héros. La norme rituelle de Sélinonte va également dans ce sens: elle précise que certains sacrifices doivent être accomplis «comme pour des héros ", d'autres " comme pour des dieux " et d'autres encore "comme pour des immortels "

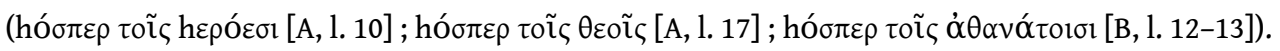
88. .ЕКROTH (2002), p. 169.

89. .VERBANCK-PIÉRARD (1989), p. 49-53.

90. .ЕКROTH (2002), p. 237.

91. .Comme à Sicyone, semble-t-il. Cf. PIRENNE-DELFORGE (2008), p. 187-190.

\section{RÉSUMÉS}

Le célèbre passage des Histoires où Hérodote distingue deux types de sacrifices en l'honneur de deux Héraclès - le dieu et le héros - (II, 44) a fait l'objet d'un grand nombre de commentaires. Considéré tantôt comme la preuve de la dualité rituelle d'Héraclès, tantôt comme une spéculation d'Hérodote, il n'a cessé de susciter la curiosité des chercheurs. Cette étude a pour objectif d'envisager, à la lumière des recherches les plus récentes, le rapport éventuel entre ce passage et le culte d'Héraclès à Thasos. Après avoir proposé une analyse philologique approfondie du passage d'Hérodote, nous tenterons d'éclaircir la signification d'une inscription toujours problématique à ce jour, le règlement du culte d'Héraclès Thasios.

A well-known passage of the Histories (2.44) where Herodotus makes a distinction between two kinds of sacrifices for Herakles - both the god and the hero - has been the focus of much commentary. Sometimes regarded as the proof of the ritual duality of Herakles, sometimes as speculation on the part of Herodotus, this passage continues to stimulate scholarly interest. This study aims to consider, in the light of the latest research, the possible link between this passage and the cult of Herakles on Thasos. Having offered a careful philological analysis of the passage in question, it attempts to clarify the meaning of a still problematic inscription, the ritual regulation of Herakles Thasios. 
AUTEUR

ZOÉ PITZ

Aspirante F.R.S.-F.N.R.S.

Université de Liège

Département des Sciences de l'Antiquité

Place du 20-Août, 7

$\mathrm{BE}$ - 4000 Liège

z.pitz@ulg.ac.be 